\title{
63 ROAD TRAFFIC INJURIES IN URBAN PAKISTANI CHILDREN AND ADOLESCENTS AGE, GENDER AND ROAD USER PERSPECTIVES
}

doi:10.1136/injuryprev-2012-040590d.63

\footnotetext{
${ }^{1,3}$ Khan Uzma Rahim, ${ }^{1}$ Razzak Junaid, ${ }^{1,3}$ Jooma Rashid, ${ }^{2}$ Laflamme Lucie. ${ }^{1}$ Department of Emergency Medicine, Aga Khan University, Pakistan; ${ }^{2}$ Karolinska Institutet, Sweden; ${ }^{3}$ Road Traffic Injury Research and Prevention Centre, Jinnah Postgraduate Medical Centre, Karachi 75510, Pakistan
}

Background More than 9 in 10 children deaths in the road traffic environment occur in low and middle countries. Little is known about the demographic distribution of those deaths and the categories of road users at risk by age and gender. This study sheds light on those issues in one large urban setting of Pakistan.

Methods This is register-based cross-sectional study in which we extracted all injuries sustained by children aged 1-19years ( $n=32$ 814) from hospital based injury surveillance system (five participating hospitals) in the city during 2007-2010. The injury distributions by category of road users was examined considering age group (1-4, 5-9, 10-14 and 15-19 years), gender and injury outcome (discharge, admission and death). We considered both vulnerable road users (pedestrians, drivers and passengers of two wheelers, drivers) and protected one (passengers of three or more wheelers).

Results Whereas RTIs among boys increased with age, they peaked among girls at age 5-9 years, for a global male to female ratio of 6 : 1. Children were most often injured as vulnerable road users. In early age groups, children were injured mostly as pillion riders (1-4 years) and as pedestrians (5-9 and 10-14 years) while in older age group (15-19 years), girls as motorcycle passengers and boys, as motorcycle drivers. Pedestrians' injuries had highest admissions $(38 \%)$ and deaths $(46 \%)$ in children. 
Conclusion At all ages, boys are more injured in road traffic crashes than girls. Both genders are at higher risk as vulnerable road users. 